$$
\bar{n}_{\lambda}=\frac{\stackrel{(\text { Bose })}{r x_{\lambda}}}{1-r x_{\lambda}}-\frac{1}{2 F_{2}^{2}}\left\{\left(2 F_{2}-F_{3}\right) \frac{r x_{\lambda}}{\left(1-r x_{\lambda}\right)^{2}}+F_{2} \frac{r x_{\lambda}+\left(r x_{\lambda}\right)^{2}}{\left(1-r x_{\lambda}\right)^{3}}\right\}+O\left(F_{2}^{-3 / s}\right) .
$$

In der früheren Arbeit, vergl. dort $(21)^{+}$, hatte sich nur

$$
\bar{n}_{\lambda}=\frac{r x_{\lambda}}{1-r x_{\lambda}}-\frac{1}{2 F_{2}} \frac{r x_{\lambda}+\left(r x_{\lambda}\right)^{2}}{\left(1-r x_{\lambda_{\lambda}}\right)^{3}}
$$

ergeben.

Bildet man aus (9) $\sum_{\lambda} \bar{n}_{\lambda}$, so ergibt sich bei $\mathrm{Be}-$ achtung von (4), (5) und (6) gerade $N$, wie es sein $\mathrm{mu}$.

Oberhalb der Einsteinschen Kondensationstemperatur $T_{\text {krit }}$ sind $F_{2}$ und $F_{3}$ von der Größenordnung $N$, das Korrektionsglied in (9) also von der Ordnung $1 / N$. Für $T \leqq T_{\text {krit }}$ ist die Berechnung der Zustandssumme in der in ${ }^{1}$ durchgeführten Weise aus Konvergenzgründen nicht mehr möglich. Dann wären aber ohnehin andere Methoden anzuwenden. Man darf nämlich bei diesen tiefsten Temperaturen das B o se-Gas nicht mehr als ideal betrachten, da die Wechselwirkungsenergie zwischen den Teilchen nicht mehr vernachlässigbar klein gegen ihre kinetische Energie ist.

Anmerkung: In der Arbeit ${ }^{1}$ sind folgende Druckfehler zu berichtigen: In den Formeln (15), (16) und (17) sind die Ausdrücke

$$
+\pi \sqrt{\frac{F_{2}}{2}} \text { und }-\pi \sqrt{\frac{F_{2}}{2}}
$$

oben und unten an die Integrale als Grenzen zu schreiben statt als Faktoren bzw. Summanden in den Formeln.

In Gl. (20), vierter Bruch, muß es $F_{2} v$ statt $F_{2} v$ heißen.

Gl. (21), dritter Ausdruck: Im Zähler des ersten Hauptzählerbruches muß beidemale $x_{\lambda}$ statt $x_{j}$ stehen.

\title{
Zur Deutung der bei Anregung in der Hohlkathode beobachteten Anomalie von $\mathrm{CuH}$
}

\author{
Von Lieselotte Reinebeck \\ Aus dem Kaiser-Wilhelm-Institut für Physik, Hechingen \\ (Z. Naturforschg. 2 a; 251-259 [1947]; eingegangen am 25. Oktober 1946)
}

\begin{abstract}
Bei Anregung in der $\mathrm{Sch}$ üler schen Hohlkathode tritt eine anomale Intensitätsverteilung beim $\mathrm{CuH}$ auf. Für diese Erscheinung sind zwei Deutungen möglich: 1. Als Prädissoziationseffekt, d.h. als eine Eigenschaft des angeregten CuH-Moleküls im Gasraum. und 2. als ein Effekt, der auf Oberflächeneigenschaften der Kupferkathode beruht. Die Beobachtungen des gleichen anomalen Verhaltens im zweiten höher angeregten Zustand des Kupferhydrids und der normale Intensitätsverlauf des CuD-Spektrums entscheiden nun eindeutig für die Deutung als Oberflächenprozeß. Die Möglichkeit, Oberflächeneffekte unter Einschaltung des Zerstäubungsprozesses der Entladung unverändert in der Gasphase zu beobachten, ist bisher noch nicht bekannt. Bemerkenswert ist auch das stark unterschiedliche Verhalten`der H-Verbindung gegenüber der entsprechenden D-Verbindung.
\end{abstract}

In früheren Arbeiten ${ }^{1,2}$ zeigten Schüler und Mitarbeiter, daß die Hohlkathode durch ihre Eigenschaften für Untersuchungen an Metallhydriden besonders geeignet ist. So wurden u. a. auch Versuche mit einer Kupferhohlkathode angestellt, die mit flüssiger Luft oder auch mit Was-

1 H. Schüler u. H. Gollnow, Z. Physik 108, 714 [1938].

${ }^{2}$ H. Sch üler, H. Gollnow, H. Haber, Z. Physik 111, 484 [1939]. ser gekühlt wurde. Während bei Anregung im Bogen (der auch bei den Untersuchungen von Heimer, von Frerichs und Bengtsson ${ }^{3}$ benutzt wurde) die Verteilung der Moleküle auf die Rotationsniveaus der Boltzmann-Statistik entspricht, zeigen die Aufnahmen bei Anregung mit der gekühlten Hohlkathode einen völlig davon ab-

3 A. u. T. He imer, Z. Physik 84, 222 [1933]; auch R. Frerichs, Z. Physik 20, 170 [1923] und E. Bentgsson, ebenda 229 [1923]. 
weichenden Intensitätsverlauf. Bei diesen Versuchen $^{2}$ enthielt eine mit flüssiger Luft gekühlte Entladungsröhre die Kupferhohlkathode. Argon oder Helium wurden als Trägergase verwendet. Das einer Boltzmann-Verteilung entspricht. Die Linie $P_{1}$, die von $J^{\prime}=0$ ausgeht, ist weitaus die intensivste, $P_{2}$ hat etwa noch die Hälfte und $P_{3}$ besitzt nur noch ${ }^{1 / 4}$ dieser Intensität (Abb. 1c).

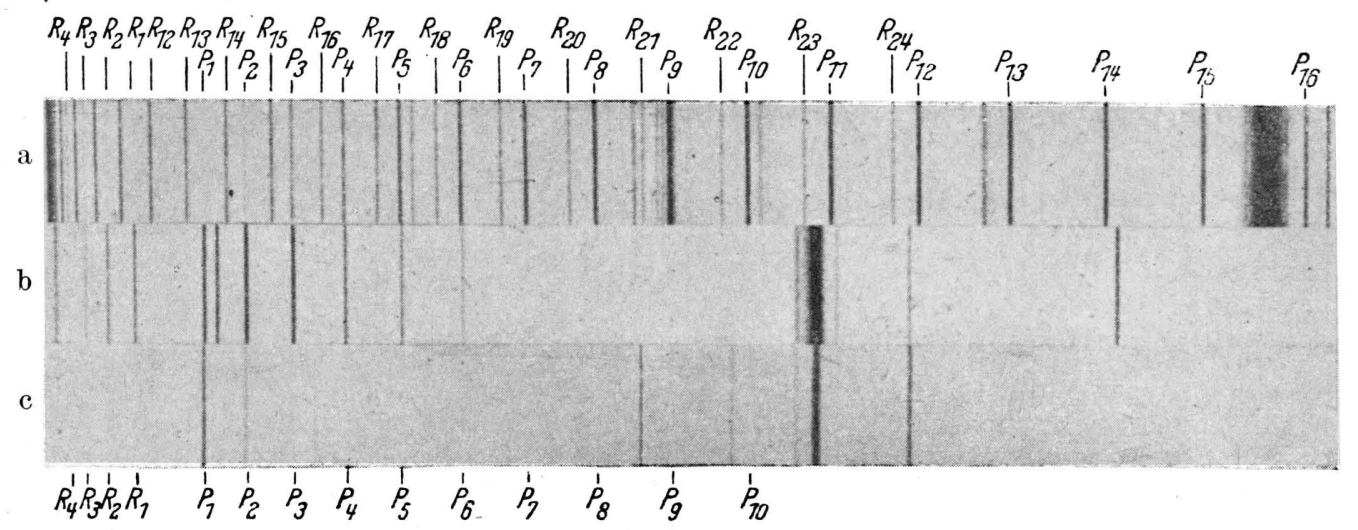

Abb. 1. CuH $\lambda$ 4280. a) Bogen b) Hohlkathodenentladung $p=2 \mathrm{~mm} \mathrm{Hg}$ c) Hohlkathodenentladung $p=0,02 \mathrm{~mm} \mathrm{Hg}$

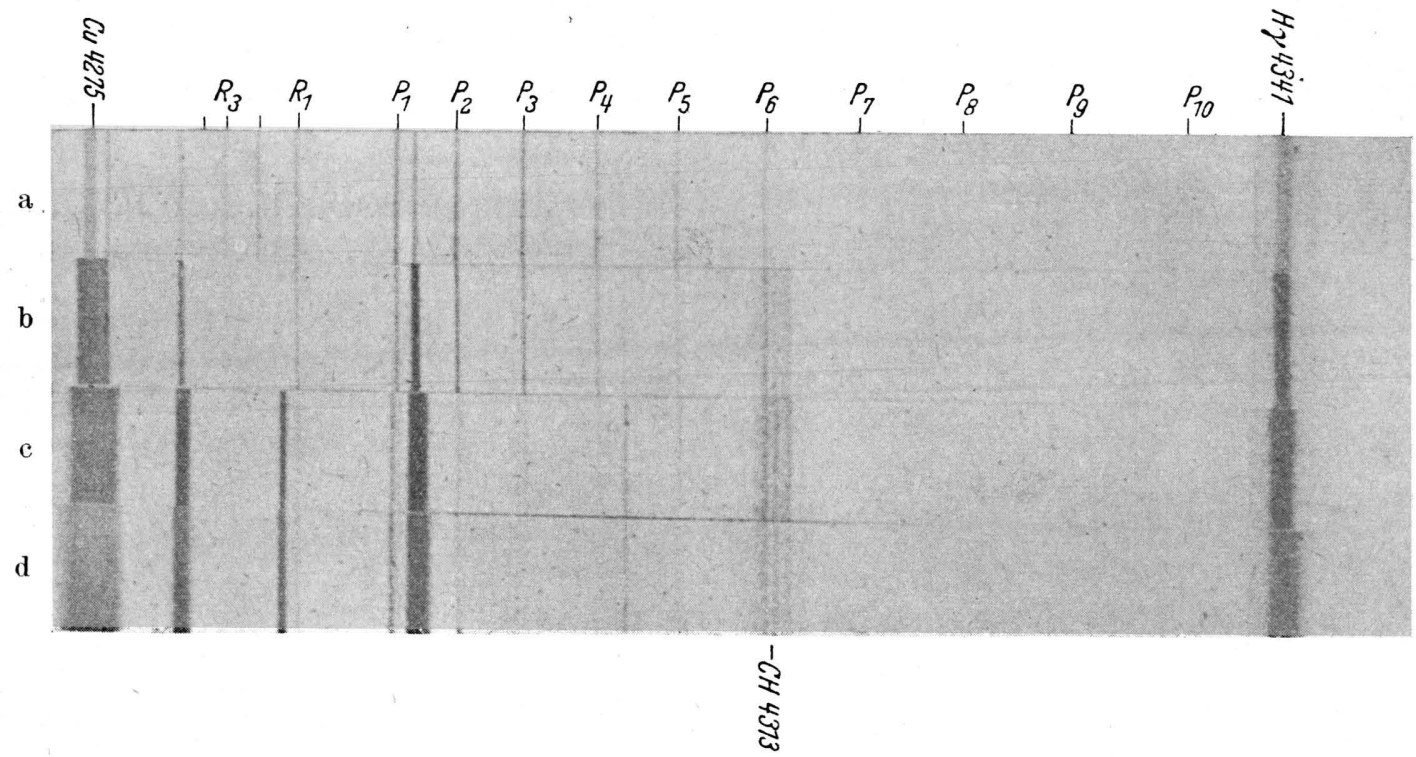

Abb. 2. Verschwinden der CuH-Bande $\lambda 4280$ bei Erhöhung der Kathodentemperatur a) Kathode mit starker Kühlung; $70 \mathrm{~mA} \mathrm{b)} \mathrm{T-Kathode,} 50 \mathrm{~mA}$ c) $T$-Kathode, $150 \mathrm{~mA}$ d) $T$-Kathode, $250 \mathrm{~mA}$

Beide Aufnahmen sind der mehrfach zitierten Arbeit von H. Schüler, H. Gollnow u. H. Haber (Z. Physik 111, 484 [1939]) entnommen.

CuH-Spektrum wurde durch Zusatz von $\mathrm{H}_{2}$ zur Entladung erzeugt und die Untersuchungen an der Bande $\lambda 4280 \AA$ durchgeführt, die die 0-0-Bande eines ${ }^{1} \Sigma^{*}{ }^{1} \Sigma$-Überganges darstellt ${ }^{3}$.

Die Versuche führten zu folgenden Resultaten:

1. Das CuH-Spektrum zeigt bei nied'rigstem Druck $(0,02 \mathrm{~mm})$ eine Intensitätsverteilung, die nicht
2. Bei Druckerhöhung des Trägergases ändert sich diese Verteilung, indem sich die Intensität der höheren Rotationslinien verstärkt (Abb. 1b u.c).

3. Bei ansteigender Kathodentemperatur, hervorgerufen durch geringere Kühlung und wachsende Stromstärke, nimmt die Intensität der gesamten $\mathrm{CuH}$-Bande ab, obgleich Kupfer- und 
Wasserstoffatome im gleichen Maße weiter zur Verfügung stehen, wie die Intensität der Linien des atomaren $\mathrm{Cu}$ und $\mathrm{H}$ zeigt (Abb.2).

Es erhebt sich nun die Frage, ob dieses Phänomen der anomalen Intensitätsverteilung beim $\mathrm{CuH}$ durch den Entladungsmechanismus in der Hohlkathode bedingt ist, oder ob eine Eigenschaft des Moleküls vorliegt.

\section{Deutung I}

G. Herzberg und L. G. Mundi $\mathrm{e}^{4}$ stellen sich auf letzteren Standpunkt und schließen, von dem Aussehen des Effektes, dem plötzlichen Intensitätsabfall nach der ersten Linie ausgehend, auf einen besonderen Fall von Prädissoziation; ein besonderer Fall deshalb, weil der Abbruch der Banden in Emission (der ein empfindlicheres Kriterium für den Beginn der Prädissoziation ist als das Einsetzen der Diffusität in Absorption) hier schon nach einer Linie erfolgt, während im allgemeinen die Prädissoziation erst bei höheren Rotationen oder erst nach einigen Schwingungen einsetzt. Liegen in einem Molekülsystem diskrete Termzustände energetisch auf gleicher Höhe mit einem Kontinuum, so treten die Zustände miteinander in Wechselwirkung. Ein Molekül, das sich in einem dieser diskreten Zustände befindet, kann strahlungslos in das Kontinuum übergehen, was einen Zerfall des Moleküls bedeutet. Für den Übergang in das Kontinuum gelten nach R. de L. Kronig ${ }^{5}$ die üblichen Auswahlregeln. Dieser Effekt wird allgemein so dargestellt, daß die diskreten Energiezustände durch Potentialkurven, die in nicht zu großer Entfernung von der Gleichgewichtslage durch eine Parabel ersetzt werden können, veranschaulicht werden, während das energetisch gleich hohe Kontinuum durch eine Kurve ohne Minimum, eine Abstoßungskürve, dargestellt wird. An dem Schnittpunkt des abstoßenden Termes mit der Potentialkurve erfolgt der strahlungslose Übergang von den diskreten $\mathrm{Zu}$ ständen in das Kontinuum, der die Dissoziation des Moleküls bedeutet. Hier beginnen in Absorption die scharfen Banden diffus zu werden, und in Emission setzt ein Intensitätsabfall ein, da von hier aus die Moleküle beide Möglichkeiten haben, sowohl strahlungslos zu zerfallen, wie mit Strahlung zum Grundzustand überzugehen. Wäh-

4 G. Herzberg u. L. G. Mundie, J. chem. Physics 8, 263 [1940]. rend im allgemeinen (z. B. beim $\mathrm{CaH}, \mathrm{NH}_{3}, \mathrm{~S}_{2}$ ) die Prädissoziation ein völliges Fehlen aller höheren Glieder der Bande bedeutet, sind beim $\mathrm{CuH}$ die Rotationslinien mit $J^{\prime} \geq 1$ noch zu sehen, wenn auch mit stark geschwächter Intensität, obgleich sie schon mit dem Kontinuum auf gleicher energetischer Höhe liegen. Herzberg und Mundie schließen daraus, daß die Wahrscheinlichkeit für strahlungslosen Zerfall nicht viel größer ist als die für Ưbergänge mit Strahlung. Das ist derselbe Schluß, zu dem sie auch bei Betrachtung des Druckeffektes gelangen (s. weiter unten).

Die beiden Verfasser führen aus, daß der abstoßende Term hier zwischen $J^{\prime}=0$ und $J^{\prime}=\mathbf{1}$ die Potentialkurve des angeregten Zustandes schneiden müßte, denn nur $P_{1}$, von $J^{\prime}=0$ ausgehend, erscheint ungestört. Weiter wird jedoch gezeigt, daß diese Annahme nicht notwendig ist, wenn der die Prädissoziation hervorrufende Term als ${ }^{3} \Sigma+$-Term angenommen wird, $\mathrm{zu}$ dem ein normales $\mathrm{Cu}$ Atom $\left({ }^{2} S\right)$ und ein normales H-Atom $\left({ }^{2} S\right)$ A'nlaß geben würden. Damit ist nach K r on ig s Auswahlregeln ${ }^{5}$ Prädissoziation nur für $J^{\prime} \geqq 1$ möglich: $P_{1}$ darf also nicht strahlungslos zerfallen, ganz gleich wo die Prädissoziationsgrenze liegt.

Ferner wird in der zitierten Arbeit darauf hingewiesen, daß auch die übrigen Erscheinungen mit Herzbergs Prädissoziationsdeutung im Einklang stehen:

Die Durchbrechung der Auswahlregeln bei Prädissoziation ${ }^{3} \Sigma \rightarrow{ }^{1} \Sigma$ ist bei schweren Molekülen bekannt.

Die Dissoziationsenergie des $\mathrm{CuH}$ im Grundzustand wird <23325 $\mathrm{cm}^{-1}$ entgegen $24300 \mathrm{~cm}^{-1}$ von der Extrapolation der Schwingungsquanten im Grundzustand; die Werte der letzteren Methode sind in der Regel aber zu hoch.

Die Intensität fällt auch bei Rotationslinien von höheren Niveaus als $J^{\prime}=1$ weiter ab, womit die Tatsache, daß bei Prädissoziation ${ }^{1} \Sigma \rightarrow{ }^{3} \Sigma$ die Utbergangswahrscheinlichkeit grob proportional zu $J^{\prime}$ ansteigt, in Übereinstimmung ist, während man sonst erwarten müßte, daß die Intensität nach der Abbruchstelle wieder zu dem gewöhnlichen thermischen Maximum ansteige.

Offen bleibt jedoch bei dieser Annahme der Prädissoziation die Druckerscheinung (CuH-Resultat 2). Deutung I bezeichnet sie als den Druckeffekt der Prädissoziation, wie er beim $\mathrm{AlH}, \mathrm{CaH}$ und $\mathrm{SrH}$ bekannt ist, was aber aus folgenden Gründen nicht ohne weiteres übernommen werden kann: die anomale Intensitätsverteilung erscheint

5 R. de L. Kronig, Z. Physik 50, 347 [1928] u. Z. Physik 62, 300 [1930]. 
bei niedrigstem Druck am deutlichsten. Bei langsamer Steigerung des Edelgasdruckes findet eine allmähliche Auffüllung der höheren Rotationsniveaus statt, die bei $2 \mathrm{~mm} \mathrm{Hg}$ bereits $\mathrm{zu}$ einer wesentlichen Änderung des Intensitätsverlaufs führt (Abb. 1bu.c). Herzberg und Mundie, bei denen die Wechselwirkungen des $\mathrm{CuH}$ mit den benachbarten Edelgasatomen im angeregten $\mathrm{Zu}$ stand erfolgen muß, meinen nun, diese Empfindlichkeit für solch geringe Druckänderungen sei ein Zeichen dafür, daß die Übergangswahrscheinlichkeit für strahlungslosen Zerfall etwa die gleiche ist wie die für Übergänge mit Strahlung. Hierbei ist nun aber zu berücksichtigen, daß bei einem Druck von $2 \mathrm{~mm} \mathrm{Hg}$ die Zeit zwischen zwei Zusammenstößen mit den Edelgasatomen in der Größenordnung von $10^{-6}$ bis $10^{-7}$ sec liegt (bei kleineren Beobachtungsdrucken ist die Zeit noch entsprechend länger), während die Lebensdauer des angeregten $\mathrm{CuH}-\mathrm{Moleküls}$ nur etwa $10^{-8} \mathrm{sec}$ betrágen dürfte. Wenn man die Erscheinung als Druckeffekt der Prädissoziation deuten wollte, wäre noch eine zusätzliche Annahme notwendig, nach der das angeregte Molekül einen sehr großen Wirkungsquerschnitt hat und für Wechselwirkungen mit benachbarten Molekülen besonders empfindlich ist.

Weiterhin läßt sich auch mit der Prädissoziationsdeutung (I) das Verhalten des $\mathrm{CuH}$ bei ansteigender Kathodentemperatur ( CuH-Resultat 3) nicht ohne weiteres erklären.

Zusammenfassend kann gesagt werden, daß es sich also um einen besonderen Fall von Prädissoziation handeln würde, der erstens nur bei geringstem Druck zu beobachten ist (sich daher bei früheren Untersuchungen, vor allem im Bogen, der Beobachtung entzog) und der zweitens durch die kritische Lage des Schnittpunktes des Abstoßungsterms in unmittelbarer Nähe des Minimus der. Potentialkurve im angeregten Zustand charakterisiert wird, so daß nur eine Linie ungestört erscheint.

\section{Deutung II}

Während also Herzberg und Mundie in den molekularen Eigenschaften des $\mathrm{CuH}$ den Anlaß für seine anomale Intensitätsverteilung suchen (Deutung I), sind Schüler und Mitarbeiter ${ }^{1,2}$ mehr geneigt, den Entstehungsprozeß des $\mathrm{CuH}$ Moleküls in der Hohlkathode als Ursache zugrunde zu legen (Deutung II).
Wenn man nun unter Aufgabe der Prädissoziation die Erscheinung von diesem Standpunkt aus diskutiert, so heißt das: Weil die anomale Verteilung der Moleküle auf die Rotationsniveaus jetzt auf den Molekülbildungsprozeß zurückgeführt wird, muß das $\mathrm{CuH}$ eine solche Verteilung schon im Grundzustand besitzen, also vor der Anregung. Wie wiederholt erwähnt, tritt die Anomalie des $\mathrm{CuH}$ bei dem bisher erreichten Druckminimum am stärksten hervor. Hier erleiden aber die Moleküle die geringste Anzahl an Zusammenstößen; die Besetzung der Rotationsniveaus wird so am wenigsten geändert sein und daher den Verhältnissen, die beim Bildungsprozeß vorliegen, am meisten entsprechen. Der Befund sagt aus, daß die erste Rotationslinie die intensivste ist; danach werden die CuH-Moleküle bevorzugt im nullten Rotationszustand gebildet. Da eine Entstehung im Gasraum durch Dreikörperstoß bei solch geringen Drucken ausgeschlossen ist, muß das $\mathrm{CuH}$ an der Oberfäche der Kathode gebildet werden. Die Beobachtungen zeigen, daß die Moleküle ohne Schwingungs-, aber mit Rotationsbehinderung entstehen; d. h. daß das Cu- und das H-Atom bei der Entstehung des $\mathrm{CuH}$ gleiche (oder entgegengesetzte) Bewegungsrichtung haben müssen. Jedes Bild, das man sich yon dem Bildungsprozeß macht, muß diesen Tatsachen entsprechen.

Die Erklärung des Druckeffektes (CuH-Resultat 2) stößt bei dieser Anschauung auf keine Schwierigkeiten. Während bei der Prädissoziationsdeutung (I) die Wechselwirkungen des $\mathrm{CuH}$ Moleküls mit den benachbarten Edelgasatomen im angeregten Zustand erfolgen müssen (s. oben), können diese bei Deutung II im Grundzustand stattfinden. Befinden sich aber die Moleküle im Grundzustand, so ist ihre Lebensdauer, die mit der Aufenthaltszeit des Moleküls im Entladungsraum gegeben ist, bedeutend länger als im angeregten Zustand, und sie haben daher die Möglichkeit, die Rotationsniveaus auch bei kleinen Drukken durch Stöße aufzufüllen.

Das Verhalten des CuH bei ansteigender Kathodentemperatur (CuH-Resultat 3) fiihrt $\mathrm{zu}$ dem Schluß, daß das Kupfer der Kathodenoberfläche sich mit einer dünnen Wasserstoffschicht überzieht, und daß es möglich ist, durch Temperatursteigerung diese zu zerstören und damit die Bildung des $\mathrm{CuH}-$ Moleküls zu hindern. 
Folgende Versuche wurden angestellt, um eine Klärung zwischen den beiden geschilderten Deutungsmöglichkeiten zu schaffen. Es wurde die mit Wasser gekühlte Entladungsröhre ${ }^{6}$ mit Kupferhohlkathode* benutzt, und wieder wurde Argon oder Helium als Trägergas verwendet. (Die Wahl des Edelgases ist dabei unwesentlich.) Zunächst wurde das $\mathrm{CuH}$ untersucht, zu dessen Erzeugung eine Probe normalen Wassers durch Abkühlung auf dem Kupfer der Kathode niedergeschlagen und dort stehen gelassen wurde, bis sich das $\mathrm{Cu}$ mit dem $\mathrm{H}$ des Wassers gesättigt hatte, was erfahrungsgemäß nach einem halben Tag mit Sicherheit eingetreten war.' Vor der Aufnahme wurde das Wasser durch Abpumpen entfernt; das Einbrennen der Kathode dauerte nur wenige Minuten, danach war das CuH-Spektrum klar auf sauberem Untergrund sichtbar.

\section{CuH-Resultate:}

4. Das so durch Einwirkung von $\mathrm{H}_{2} \mathrm{O}$ entstandene CuH-Spektrum zeigt dieselbe anomale Intensitätsverteilung (Abb.3a), wie sie aus den Untersuchungen von Schüler und Mitarbeitern bekannt ist, wo das $\mathrm{CuH}$ durch Beifügung von $\mathrm{H}_{2}$ währenḑ der Entladung entstand.

5. Treten zum reinen Elektronenübergang Schwingungen hinzu, so zeigen diese Banden den gleichen anomalen Intensitätsverlauf wie die bisher untersuchte 0-0-Bande.

Diese beiden Ergebnisse ermöglichen jedoch keine Entscheidung zwischen den zwei Deutungen, da sie durch beide Anschauungen erklärt werden können. Der Befund 4 (daß sich dieselbe Anomalie auch bei einem CuH-Molekül zeigt, das durch $\mathrm{H}_{2} \mathrm{O}$-Einwirkung entsteht) ist für die Deutung I unwesentlich, da die Prädissoziation von der Art der Molekülentstehung unabhängig ist; denn hier ist der Grundzustand normal besetzt, und die Anomalie des $\mathrm{CuH}$ kommt erst beim Übergang zum gestörten angeregten Term zustande. Für die andere Deutung (II) zeigt dieser Versuch, daß auch ein so entstandenes $\mathrm{CuH}$ Molekül unter Bevorzugung des nullten Rotationszustandes gebildet wird. - Die Erscheinung 5 der-

6 H. Schüler u. H. Gollnow, Z. Physik 93, 611 [1935]. selben Anomalie beim Auftreten von Schwingungen heißt bei der Prädissoziationsdeutung (I), daß das für $J^{\prime}=0$ bei $v^{\prime}=0$ bestehende Verbot des strahlungslosen Zerfalls in derselben Strenge für die höheren Schwingungen gilt; auch hier darf $P_{1}$ nicht prädissoziieren. Dies wurde auch von Herzberg in seiner Arbeit für die Prädissoziation gefordert. Jedoch ist es auch bei Deutung II verständlich: Wenn durch die gleiche Bewegungsrichtung des $\mathrm{Cu}$ - und des $\mathrm{H}$-Atoms der nullte Rotationszustand bei der Molekülbildung bevorzugt wird, werden außerdem unabhängig davon Schwingungen auftreten können.

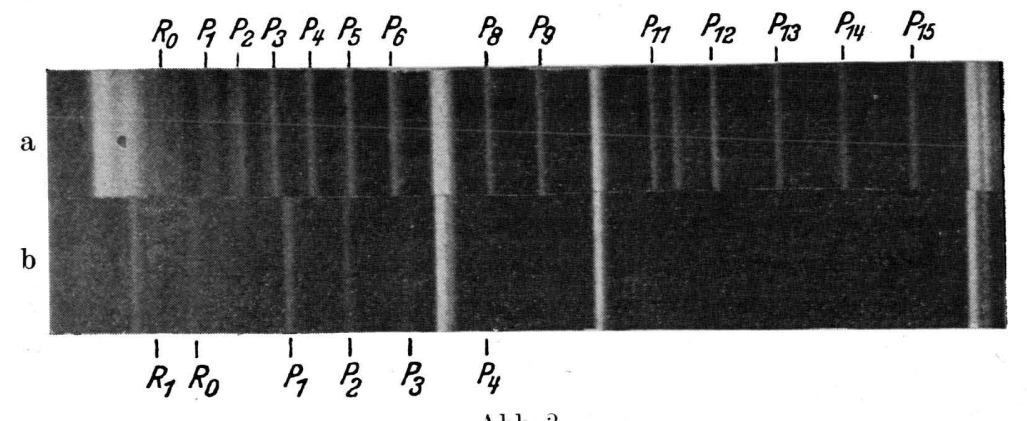

Abb. 3 .

a) CuH-Spektrum mit Ar. $50 \mathrm{~mA} ; 10 \mathrm{Min}$. belichtet; $p \sim 2 \mathrm{~mm} \mathrm{Hg}$; b) CuD-Spektrum mit Ar. $5 \mathrm{~mA} ; 20 \mathrm{Min}$. belichtet; $p \sim 2 \mathrm{~mm} \mathrm{Hg}$

Daraufhin wurden Untersuchungen mit $\mathrm{CuD}$ angestellt, da man bei vorliegender Prädissoziation für den Übergang zur deuterierten Verbindung bestimmte Anderungen im Bandenverlauf des Spektrums annehmen kann. Es wurde auf dieselbe Weise schweres Wasser auf die Kathode gebracht.

Die Versuche ergaben folgendes:

CuD-Resultate:

1. Die 0-0-Bande des $\mathrm{CuD}$ hat eine Intensitätsverteilung, wie man sie nach der BoltzmannStatistik erwarten würde. Die Feinstruktur ist bis $\mathrm{zu}$ hohen Rotationen gut sichtbar und es tritt kein Abfall der Intensität auf (Abb.3b).

2. Die CuD-Banden desselben Systems mit $v^{\prime}>0$ zeigen ebenfalls normalen Intensitätsverlauf (untersucht wurden $v^{\prime}=1$ und $v^{\prime}=2$ ).

3. Das CuD-Spektrum nimmt in ähnlicher Weise wie das $\mathrm{CuH}$ mit wachsender Kathodentemperatur an Intensität ab.

Dieses Ergebnis, daß durch den Übergang zur schwereren Verbindung das gesamte Aussehen

* Die Kathode bestand aus Elektrolytkupfer. 
eines Spektrums grundlegend geändert wird, ist überraschend und weicht von jedem der bisher bekannten Isotopieeffekte bei Molekülen ab.

Im allgemeinen bewirkt die Einführung eines Isotops in eine Verbindung vor allem eine Verschiebung der Schwingungsniveaus (Schwingungsisotopieeffekt) und der Rotationsniveaus (Rotationsisotopieeffekt), deren Auftreten durch das veränderte Trägheitsmoment des Moleküls bedingt ist. Ferner sind auf Grund der verschiedenen Nullpunktsenergien auch die Nullinien der 0-0-Banden der beiden isotopen Moleküle gegeneinander verschoben. (Da es sich hier um die reinen Elektronenübergänge $\nu_{0}$ handelt, spielt weder der Schwingungs- noch der Rotationsisotopieeffekt eine Rolle.) Diese Nullpunktsenergie oder Nullpunktsschwingung ist in allen experimentellen Beobachtungen enthalten und verbleibt als Restenergie auch bei der Schwingungsquantenzahl $v=0$ und der Rotationsquantenzahl $\boldsymbol{J}=0$, da kein Molekül in absoluter Ruhe existiert. Zieht man nunmehr die Nullpunktsenergien von den empirisch gewonnenen Termen ab, d. h. geht man $\mathrm{zu}$ einer hypothetischen Schwingung von $v=-1 / 2$ über, so erhält man die theoretische 0-0-Bande, die den Elektronensprung $\nu_{e}$ ohne Schwingung und ohne Rotation zwischen den ursprünglichen Termen bedeutet und deren Terme beim Ubergang zur schwereren Verbindung praktisch unverändert bleiben. Die geringfügige Verschiebung, die auch hier noch auftreten kann, ist - analog der Isotopenverschiebung bei Atomspektren - auf die Elektronenbewegung zurückzuführen. Auf das Elektron, das angeregt wird, wird durch die Massenänderung eine etwas andere Kraft wirken. Im allgemeinen beträgt diese Verschiebung einige Hundertstel eines $\mathrm{cm}^{-1}$, nur bei den Wasserstoffverbindungen werden durch die Verdoppelung der H-Massen Elektronenisotopieeffekte bis zu $26 \mathrm{~cm}^{-1}$ beobachtet?. Die Größe dieses Effektes (auch bei den $\mathrm{H}$-Verbindungen) kann aber gegenüber den absoluten Lageänderungen, die von der Nullpunktsverschiebung verursacht sind, vernachlässigt werden.

Das $\mathrm{CuD}$ - das von M. Jeppeson ${ }^{8}$ (auch von T. Heimer ${ }^{9}$ ) analysiert ist - weist eine Elektronenisotopenverschiebung von $19 \mathrm{~cm}^{-1}$ auf; $\mathrm{d}$. h.

7 H. Sponer, Molekülspektren und ihre Anwendung auf chemische Probleme, S. 162, Verlag J.Sp r in ger, Berlin 1936.

8 M. Jeppes on, Physic. Rev. 50, 445 [1936].

9 T. Heimer, Naturwiss. 23, 372 [1935]. die Differenz der angeregten Zustände bei $\mathrm{CuH}$ und $\mathrm{CuD}$ nach Abzug der Nullpunktsenergien beträgt.19 $\mathrm{cm}^{-1}$ (Abb. 4).

In Abb. 4 sind die Nullinien $\nu_{0}$ der 0-0-Banden und die zugehörigen Terme - alle beobachtbaren Größen also - ausgezogen gezeichnet. Der theoretische Elektronensprung $\nu_{e}$ nach Abzug der Nullpunktsenergien und die ursprünglichen Terme, deren Differenz er darstellt - kurz alle rechnerischen Größen -, sind dagegen als gestrichelte Geraden oder Pfeile dargestellt.

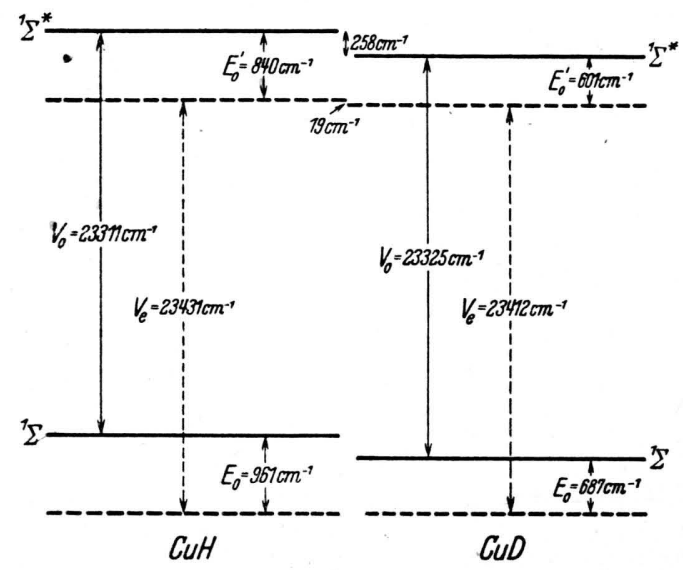

Abb. 4.

Sichtbar (ausgezogen) ist der Elektronenübergang $\mathbf{v}_{\mathbf{0}}$, in dem die Nullpunktsenergien noch enthalten sind; er beträgt bei $\mathrm{CuH} \quad v_{0}=23311 \mathrm{~cm}^{-1}$, bei $\mathrm{CuD}$ $v_{0}=23325 \mathrm{~cm}^{-1}$. Die $0-0$-Bande des $\mathrm{CuD}$ ist also gegenüber der des CuH-Spektrums nach Violett verschoben, und der Übergang scheint mehr Energie zu benötigen als beim $\mathrm{CuH}$. Jedoch erhält man durch die beobachtbaren Größen nur relative Werte, und man muß, um die Schemata der beiden isotopen Verbindungen gegeneinander festzulegen, zu den ursprünglichen Termen und zur theoretischen 0-0-Bande $v_{e}$ übergehen. Die Lage der Terme der beiden Verbindungen wird dadurch fixiert, daß man für beide den gleichen absoluten Grundzustand (nach Abzug der Nullpunktsenergien) wählt. Es ist berechtigt, für beide isotopen Moleküle, die sich in absoluter Ruhe befinden, die gleiche Energie anzunehmen, denn für isotope Moleküle ist auch die Dissoziationsenergie die gleiche, d.h. die Bindungskräfte zwischen den Atomen sind gleich groß ${ }^{\mathbf{1 0}}$. Vom absoluten Grundterm aus erfolgt der Elektronensprung $v_{e}$ (die theoretische 0 -0-Bande), der bei $\mathrm{CuH} v_{e}=23431 \mathrm{~cm}^{-1}$, bei $\mathrm{CuD}$ dagegen $v_{e}=23412 \mathrm{~cm}^{-1}$ beträgt. Nach Abzug der Nullpunktsenergien liegt der angeregte Term des CuD also um $19 \mathrm{~cm}^{-1}$ nach kleineren Energien zu verschoben gegenüber dem des CuH. Der sichtbare Elektro-

10 G. H e r z b e r g, Molekülspektren und -struktur, S. 300, Verlag Th. St e in k o p ff, Dresden und Leipzig. 
nenübergang $v_{0}$ ist beim $\mathrm{CuD}$ deshalb größer, weil hier in der Termdifferenz noch die Nullpunktsenergien enthalten sind und diese bei der schweren Verbindung sowohl im Grundzustand als auch im angeregten Zustand kleiner sind als bei der leichten Verbindung [Grundzustand: $E_{\mathbf{0}}(\mathrm{CuH})=961 \mathrm{~cm}^{-1}$; $E_{0}(\mathrm{CuD})=687 \mathrm{~cm}^{-1} ;$ angeregter Zustand: $E_{0}{ }^{\prime}(\mathrm{CuH})$ $\left.=840 \mathrm{~cm}^{-1} ; E_{0}{ }^{\prime}(\mathrm{CuD})=601 \mathrm{~cm}^{-1}\right]$ und hierdurch die Differenz zwischen den Termen größer wird. Ausschlaggebend für den Energiezustand ist jedoch die absolute Lage. Der beobachtete angeregte Term ( ${ }^{1} \Sigma^{*}$ ausgezogen gezeichnet) enthält, vom gemeinsamen Grundzustand ( ${ }^{1} \Sigma$ gestrichelt gezeichnet) aus gerechnet, die absolute Energie: reiner Elektronensprung $\boldsymbol{v}_{\boldsymbol{e}}+$ Nullpunktsenergie des angeregten Zustandes $\boldsymbol{E}_{0}{ }^{\prime}$ (bzw. Nullpunktsenergie des Grundzustandes $E_{0}+$ experimentelle 0-0-Bande $v_{0}$ ). $v_{e}+E_{0}{ }^{\prime}=E_{0}+v_{0}=$ absolute Energie des ${ }^{1} \Sigma^{*}$ beob. Das ist bei $\mathrm{CuH}^{1}{ }^{1} \Sigma^{*}$ beob. $=23431$ $\left(\nu_{e}\right)+840\left(E_{0}^{\prime}\right)=24271 \mathrm{~cm}^{-1}$ abs. Energie und bei $\mathrm{CuD}{ }^{1} \Sigma^{*}$ beob. $=23412\left(v_{e}\right)+601\left(E_{0}{ }^{\prime}\right)=24013 \mathrm{~cm}^{-1}$ abs. Energie. Durch den beiden Verbindungen gemeinsamen absoluten Grundzustand ergibt sich also die Differenz der beobachteten angeregten Terme zu $258 \mathrm{~cm}^{-1}$. Bei einem abstoßenden Term kann yon einer Nullpunktsenergie nicht mehr gesprochen werden, da diese erst in Erscheinung tritt, wenn die Potentialkurve wenigstens in der Nähe der Gleichgewichtslage durch eine Parabel angeglichen werden kann. Nur bei Kurven mit einem ausgesprochenen Minimum existieren diskrete Schwingungen, und nur da können auch Nullpunktsschwingungen auftreten, während auf dem Abstoßungsterm eine Nullpunktsschwingung, die dem Molekül als Garıes gehören soll, ihren Sinn verliert.

Bei dem Vorschlag, die Intensitätsverteilung des $\mathrm{CuH}$ mit Prädissoziation (I) zu deuten, müßte also der $3 \Sigma$-Term, der zur Prädissoziation führt, auch beim Übergang zum $\mathrm{CuD}$ unverschoben erscheinen, bis auf den geringfügigen Elektronenisotopeneffekt von $19 \mathrm{~cm}^{-1}$. Schneidet der Abstoßungsterm beim $\mathrm{CuH}$ ungefähr in der Höhe von $v^{\prime}=0$ und $J^{\prime}=0$, so muß dieser beim $\mathrm{CuD}$ bei höheren Rotationen, auf jeden Fall aber bei $v^{\prime}=1$ schneiden. Wie Abb. 4 zeigt, beträgt die Differenz zwischen der absoluten Lage des Abstoßungsterms und dem Minimum der CuD-Potentialkurve $258 \mathrm{~cm}^{-1}$; demnach müßte der Intensitätsabfall etwa bei $J^{\prime}=9$ erwartet werden, und das erste Schwingungsniveau (mit $v^{\prime}=1$ ) würde mit einem Abstand von $1148 \mathrm{~cm}^{-1}$ von der 0-0-Bande sicher über der Prädissoziationsgrenze liegen. Die Aufnahmen (CuD-Resultat 2) beweisen aber eindeutig, daß die 0 -0-Bande des $\mathrm{CuD}$ bis $J^{\prime}=25$ keinen Intensitätsabbruch zeigt, und daß auch bei $v^{\prime}=1$ und $v^{\prime}=2$ die Banden keine Intensitätsstörung auf. weisen. Wenn man das verschiedene Verhalten bei
$\mathrm{CuH}$ und $\mathrm{CuD}$ damit erklären wollte, daß beim letzteren der Abstoßungsterm die Potentialkurve des angeregten Zustandes nicht mehr schneidet, sondern tiefer vorbeigeht, so müßte man hier die Existenz der Nullpunktsenergien leugnen!

Die von Herzberg diskutierte Deutung kann also mit den jetzigen Befunden nicht in Einklang gebracht werden, und man muß daher die anomale Intensitätsverteilung beim $\mathrm{CuH}$ auf die Molekülentstehung (Deutung II) zurückführen.

Darüber hinaus gibt es noch einen weiteren Befund ( $\mathrm{CuH}$-Resultat 6 ), der eindeutig gegen die Erklärung der Anomalie als Prädissoziationseffekt spricht. Es handelt sich um Untersuchungen an einem zweiten $\mathrm{CuH}$-Übergang $\left({ }^{1} \Sigma^{* *}-{ }^{1 \Sigma}\right)$, der

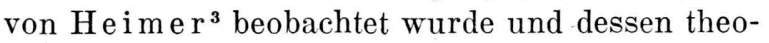
retische 0-0-Bande $\nu_{e}$ bei $26369 \mathrm{~cm}^{-1}$ liegt.

\section{Die Aufnahmen zeigen:}

CuH-Resultat 6: Sowohl bei der 0-1-Bande wie bei der ebenfalls beobachteten 1-2-Bande des ${ }^{1} \Sigma^{* *}-1 \Sigma$-Überganges liegt die gleiche anomale Intensitätsverteilung vor wie bei den Banden des Überganges ${ }^{1} \Sigma^{*}-{ }^{1} \Sigma$.

Die Annahme, daß auch bei diesem zweiten angeregten Zustand Prädissoziation vorliegen sollte, ist äußerst unwahrscheinlich, denn auch hier müßte der Abstoßungsterm gerade an derselben kritischen Stelle schneiden wie beim ${ }^{1} \Sigma^{*}-{ }^{1} \Sigma$-Übergang. Er müßte ebenfalls ein ${ }^{1} \Pi$ oder ${ }^{3} \Sigma$-Term sein, damit nur gerade das Niveau $J^{\prime}=0$ nicht prädissoziieren darf, und sollte alle Eigenschaften besitzen, wie sie von dem von Herzberg vorgeschlagenen schneidenden Term gefordert wurden. Bisher ist in der Spektroskopie noch kein Fall bekannt, in dem die Prädissoziation schon nach einer Linie einsetzt, und es ist nicht anzunehmen, daß diese Art von Prädissoziation zufällig bei zwei verschieden angeregten Zuständen des $\mathrm{CuH}$ in genau der gleichen Weise auftreten sollte. Dagegen bildet der Befund einen Beweis für die andere Deutung (II) der CuH-Anomalie durch die Molekülbildung. Die gleiche Intensitätsverteilung bei beiden Übergängen spricht eindeutig für eine Anomalie der Besetzung der Rotationsniveaus im gemeinsamen Grundzustand, die aber nur durch Molekülbildung an der Metalloberfläche erklärt werden kann. Die beobachteten Banden von ${ }^{1} \Sigma^{* *}-1 \Sigma$ zeigen genau das Verhalten, das von der Deutung II a priori gefordert wird. 
Die Auffassung, daß eine Molekülbildung an der Kathodenoberfläche stattfinden soll, wurde bisher vor allem von E. $\mathrm{Hu}$ l th én ${ }^{11}$ abgelehnt, aber die Intensität der Banden auch bei geringstem Trägergasdruck und damit noch bedeutend kleinerem Metalldampfdruck zeigt, daß eine Entstehung durch Dreierstoß im Gasraum unmöglich ist, und man muß also zwangsweise den Entstehungsprozeß an der Kathodenoberfläche annehmen. Auch wird in physikalisch-chemischen Arbeiten (s. Eucken ${ }^{12}$ S. 1257) gezeigt, daß durch Adsorption Oberflächenverbindungen entstehen können; insbesondere ist eine Hydridbildung durch Adsorption von H-Atomen an Kupfer oder Nickel bekannt ${ }^{13,14}$.

Der vorliegende Bildungsprozeß, ganz gleich wie man ihn im einzelnen annehmen will, muß folgende Punkte erfüllen:

a) Das $\mathrm{CuH}-$ Molekül muß unter Bevorzugung der nullten Rotation und ohne Schwingungsbehinderung entstehen; es muß also die anomale Intensitätsverteilung im Grundzustand besitzen.

b) Beim $\mathrm{CuD}$ dagegen sollen alle Rotationen und Schwingungen praktisch ungestört erscheinen.

c) Es darf sich keine Änderung der Intensitätsverteilung zeigen, ob das Molekül durch $\mathrm{H}$ Atome oder durch $\mathrm{H}_{2} \mathrm{O}$ gebildet wird.

d) Versuche mit Aluminium ergaben, daß hier keine solche Änderungen im Intensitätsverlauf vorhanden sind, wenn man zur deuterierten Verbindung übergeht. (Auch hier wurden die Spektren durch Einwirkung von $\mathrm{H}_{2} \mathrm{O}$ bzw. $\mathrm{D}_{2} \mathrm{O}$ erzeugt.)

Es erhebt sich nun die Frage, ob das verschiedene Verhalten der $\mathrm{H}$ - und der D-Verbindungen auf das Kupfer beschränkt ist, und wie man sich dieses Benehmen erklären kann. Da die Anregung bei den Untersuchungen am AlH bzw. AlD unter denselben Versuchsbedingungen durchgeführt wurde wie an der Kupferkathode, und hier kein verschiedenes Verhalten der beiden Verbindungen beobachtet wurde, liegt der Schluß nahe, daß es sich beim $\mathrm{Cu}$ um eine besondere Wirkung von Oberflächenkräften handelt. Nun können Ober-

11 E. Hulthén, Ark. Mat., Astron. Fysik, Ser. B 27, Nr. 5 .

${ }_{12}$ A. Eucken, Lehrbuch der chem. Physik, Bd. II, 2, Akademische Verlagsgesellschaft Becker \& Erle r, Leipzig. flächenkräfte bei Katalysatoren ganz verschiedene Wirkungen haben, und die verschiedene Wirkungsweise auf die beiden Wasserstoffisotope müßte eine spezielle Eigenschaft des Kupfermetalls sein. Weiterhin ist die Bildung von elektrischen Doppelschichten an Metalloberflächen bekannt, an denen Adsorption stattgefunden hat. Die durch Adsorption an Metalloberflächen gebildeten Moleküle sind stark polarisiert, zum Teil so stark, daß die oberste Schicht als Ion zurückbleibt (siehe Eucken ${ }^{12}$ S. 1317). Vielleicht ist in diesen Befunden die Parallele zu suchen zu dem CuH-Molekül, das ohne Rotation, aber mit Schwingungen entstehen soll.

Eine ähnliche Erscheinung, wie sie beim $\mathrm{CuH}$ beim niedrigsten Druck auftritt, scheint bei Mrozowski ${ }^{15}$ angedeutet $\mathrm{zu}$ sein. Nachdem Schüler und Mitarbeiter in ihrer CuH-Deutung (II) auf die Rolle der Oberfläche bei der Intensitätsverteilung hingewiesen hatten, findet $\mathrm{Mro-}$ $\mathrm{z}$ ow ski bei seinen Untersuchungen am $\mathrm{HgH}$ eine Anderung der Intensitätsverteilung je nach dem Kathodenmaterial, das er verwendet. Während er den Intensitätsverlauf bei einer Kupferkathode als „,normal" bezeichnet, beobachtet er bei einer Eisenkathode eine Verteilung, die ,einer extrem niedrigen Temperatur" entspricht. Nähere Angaben über dieses Verhalten sind in der Arbeit nicht enthalten.

Interessant ist weiterhin der Vorgang des Austausches zwischen den $\mathrm{H}$ - und den D-Atomen beim Stehenlassen der Flüssigkeit über der Kathode. Ist die Kathode z. B. mit D-Atomen gesättigt und man läßt $\mathrm{H}_{2} \mathrm{O}$ darüber stehen, so tauschen sich die beiden Isotope aus, und man bekommt statt eines Deutrid- ein Hydrid-Spektrum. Dieses gilt sowohl bei einer Al- wie bei einer $\mathrm{Cu}$-Kathode. Läßt man dagegen gasförmigen $\mathrm{H}_{2}$ über einer mit $\mathrm{D}$ gesättigten Aluminiumkathode stehen, so findet in der gleichen Zeit kein merkbarer Austausch statt; das AlD-Spektrum erscheint mit derselben Intensität weiter. Der Grund für die Wechselwirkungen der Metallatome mit dem Wasserstoff der flüssigen Verbindung und für das Ausbleiben des Austausches beim $\mathrm{H}_{2}$ läßt sich bisher nicht angeben. Dagegen scheint das verschiedene Verhalten des $\mathrm{Cu}$ gegenüber $\mathrm{H}$-Atomen (die beim Zulassen von $\mathrm{H}_{2}$

${ }^{13} \mathrm{E}$. Pietsch, Z. Elektrochem. angew. physik. Chem. 39, 577 [1933].

$14 \mathrm{O}$. Th. Leipunski, Acta physicochim. URSS 2, 737 [1935].

15 S. Mrozowski, Physic. Rev. 58, 332 [1940]. 
während der Entladung entstehen) und dem gasförmigen $\mathrm{H}_{2}$ (der ohne Entladung über der Kathode stehen gelassen wurde) wiederum in physikalisch-chemischen Arbeiten ein Analogon zu haben. Es können aus einer verdünnten Gasphase auf Metalle (als Beispiele sind $\mathrm{Cu}$ und $\mathrm{Ni}$ genannt) aufprallende H-Atome zu einer Hydridbildung führen, während die Metalle molekularem $\mathrm{H}_{2}$ gegenüber keinerlei Neigung zur Hydridbildung zeigen (s. Eucken ${ }^{12}$ S. 1447).

Schließlich bildet noch der „Schwundeffekt“, das Verhalten bei ansteigender Kathodentemperatur, eine Differenzierung, die erst durch weitere Experimente geklärt werden kann. Während bei steigender Kathodentemperatur die Intensität der $\mathrm{AlH}-$ und AlD-Spektren ansteigt (bei AlH scheinbar in stärkerem Maße als bei AlD), sinkt sie bei den Spektren des $\mathrm{CuH}, \mathrm{CuD}$ und $\mathrm{BiH}$ ab. Beim $\mathrm{Wismut}^{16}$ sind die BiH-Banden bereits bei Wasserkühlung praktisch nicht mehr zu sehen, während sie bei Kühlung mit flüssiger Luft unter sonst gleichen Entladungsbedingungen (vor allem bei gleicher Menge von atomarem $\mathrm{Bi}$ und $\mathrm{H}$ und gleicher Belichtungszeit) stark hervortreten. Beim

${ }^{16}$ H. Schüler u. H. Haber, Z. Physik 112, 614 [1939].

17 Gauger u. Taylor, J. Amer. chem. Soc. 45, 920 [1923]; v. Hemptinne, Z. physik. Chem. 27, 429 [1898]; Nikitin, J. Russ. Phys. Chem. Soc. 58, $1081[1926]$.
Kupfer ist die Schwächung des Hydridspektrums beim Übergang zur Wasserkühlung noch nicht merkbar, setzt aber ein, wenn die Kathode weniger gekühlt ist, das $\mathrm{Cu}$ der Kathode sich aber noch weiter unter Rotglut befindet. Hier sei nochmals auf die erwähnte Arbeit von E. Pietsch ${ }^{13}$ hingewiesen, in der das Kupfer als besonders temperaturempfindlich bezeichnet und festgestellt wird, daß höhere Temperatur den Zerfall des $\mathrm{CuH}$ begünstigt bzw. dessen Entstehung hindert; auch bei $\mathrm{Pt}, \mathrm{Pd}$ und $\mathrm{Ni}^{17}$ wird verstärkter Zerfall der Oberflächenmoleküle mit wachsender Temperatur beobachtet.

Die hier diskutierten Befunde an Hohlkathoden werden dann erst ihren vollen Beitrag zum Studium von Oberflächenprozessen liefern können, wenn man sich darüber klar geworden ist, was der Zerstäubungs-Verdampfungs-Prozeß an der Kathode, der zwischen dem eigentlichen Oberflächenprozeß und der spektroskopischen Beobachtung immer eingeschaltet ist, für eine Rolle spielt. Die bisherigen Befunde am $\mathrm{CuH}$ deuten jedenfalls darauf hin, daß der Zerstäubungs-VerdampfungsProzeß auf die Untersuchung des Oberflächenprozesses nicht störend wirkt.

Abschließend möchte ich Hrn. Prof. Dr. Schüler danken für sein großes und förderndes Interesse an dieser Arbeit, für die großzügige Bereitstellung von Apparaturen sowie für die Möglichkeit, mich an seinen spektroskopischen Untersuchungen zu beteiligen.

\title{
Ein einfaches Näherungsverfahren zur quantenmechanischen Behandlung der $\pi$-Elektronensysteme aromatischer Kohlenwasserstoffe I
}

\author{
Von Hermann Hartmann \\ Aus dem Institut für physikalische Chemie der Universität Frankfurt a. M. \\ (Z. Naturforschg. 2 a, 259-262 [1947]; eingegangen am 18. November 1946)
}

\begin{abstract}
Es wird ein quantenmechanisches Näherungsverfahren entwickelt, das die Wechselwirkungsenergie der $\pi$-Elektronen im Grundzastand aromatischer Kohlenwasserstoffe praktisch ohne Rechenarbeit aus der Strukturformel abzulesen gestattet. Die Resultate des Verfahrens sind im großen und ganzen ebensogut mit der Erfahrung in Übereinstimmung, wie die Resultate des Slater-Paulingschen Verfahrens. Im Falle des Stoffpaares Anthracen-Phenanthren sind sie den Resultaten des Slater-Paulingschen Verfahrens überlegen. Mit Hilfe des Verfahrens lassen sich die Wechselwirkungsenergien der $\pi$-Elektronen für ganze Stoffklassen überblicken. Einige Beispiele hierzu werden mitgeteilt.
\end{abstract}

$\mathbf{B}^{\mathrm{e}}$ i der Behandlung der $\pi$-Elektronensysteme aromatischer Kohlenwasserstoffe mit dem Hückelschen ${ }^{1}$ oder dem Slater-Paulingschen ${ }^{2}$

1 E. Hückel, Z. Physik 70, 204 [1931]. Hier ist das erste Hü ckelsche Verfahren gemeint, von dem sich die späteren Paulingschen Rechnungen nur formal unterscheiden.
Näherungsverfahren treten, wenn es sich um große Moleküle handelt, rechnerische Schwierigkeiten auf. Wenn die Moleküle Symmetrie-Elemente besitzen, ist zwar mit gruppentheoretischen Methoden ${ }^{3}$ eine

2 L. Pauling, J. chem. Physics 1, 280 [1933].

3 R. Mulliken, Physic. Rev. 43, 279 [1933]. 